\title{
Changes in whole-blood PUFA and their predictors during recovery from severe acute malnutrition
}

\author{
Esther Babirekere-Iriso ${ }^{1,2 *}$, Charlotte G. Mortensen ${ }^{2}$, Ezekiel Mupere ${ }^{3}$, Maren J. H. Rytter ${ }^{2}$, \\ Hanifa Namusoke ${ }^{1}$, Kim F. Michaelsen ${ }^{2}$, André Briend ${ }^{2}$, Ken D. Stark ${ }^{4}$, Henrik Friis ${ }^{2}$ and Lotte Lauritzen ${ }^{2}$ \\ ${ }^{1}$ Mwanamugimu Nutrition Unit, Department of Paediatrics, Mulago Hospital, PO Box 7051, Kampala, Uganda \\ ${ }^{2}$ Department of Nutrition, Exercise and Sports, Faculty of Science, University of Copenhagen, 1958 Frederiksberg C, Denmark \\ ${ }^{3}$ Department of Paediatrics, Makerere College of Health Sciences, PO Box 7072, Kampala, Uganda \\ ${ }^{4}$ Department of Kinesiology, University of Waterloo, 200 University Avenue, Waterloo, ON, Canada N2L $3 G 1$
}

(Submitted 24 July 2015 - Final revision received 17 December 2015 - Accepted 4 February 2016 - First published online 21 March 2016)

\section{Abstract}

Children with severe acute malnutrition (SAM) with complications require in-patient management including therapeutic feeding. Little attention has been given to the effects of these feeds on the essential fatty acid status of children with SAM. The objective of this study was to describe changes in the PUFA composition in whole blood in children with SAM during treatment and to determine predictors of change. This prospective study took place in a paediatric nutrition rehabilitation unit in Kampala, Uganda, and assessed whole-blood fatty acid composition of children with SAM at admission, transition, discharge and follow-up ( 8 and 16 weeks). ANCOVA was used to identify predictors of change in whole-blood PUFA. The study included 120 children with SAM and twenty-nine healthy control children of similar age and sex. Among the SAM children, $38 \%$ were female and $64 \%$ had oedema. Whole-blood $n$-6 PUFA proportions increased from admission to follow-up, except for arachidonic acid, which decreased by $0.79(95 \%$ CI $0.46,1.12)$ fatty acid percentage (FA\%) from admission to transition and $0.10(95 \%$ CI 0.23 , $0.44) \mathrm{FA} \%$ at discharge. $n$-3 Long-chain (LC) PUFA decreased by 0.21 (95\% CI 0.03, 0.40) FA\% at discharge and 0.22 (95\% CI $0.01,0.42) \mathrm{FA} \%$ at 8 weeks of follow-up. This decrease was greater in children from families with recent fish intake and those with nasogastric tube feeding. Current therapeutic feeds do not correct whole-blood levels of LCPUFA, particularly $n-3$ LCPUFA, in children with SAM. Increased attention is needed to the contents of $n-3$ LCPUFA in therapeutic feeds.

Key words: PUFA: Severe acute malnutrition: Recovery: Children

Severe acute malnutrition (SAM) is a major health problem in children in low-income and middle-income countries ${ }^{(1)}$. Children with complicated SAM require in-patient management of presenting complications while therapeutic feeds are being initiated. The standard nutritional treatment is milk-based formulas, such as starter F-75, initiated as soon as possible after admission $^{(2)}$. F-75 is designed to restore physiological and metabolic functions and electrolyte balance and to maintain body weight. After stabilisation, the feed is gradually changed to a formula (F-100) that provides sufficient energy and protein to support rapid catch-up growth or directly to ready-to-use therapeutic feed (RUTF) ${ }^{(3)}$. RUTF is typically continued after discharge and given at regular follow-ups until recovery. An average period of 8 weeks of nutritional rehabilitation is considered adequate for recovery ${ }^{(4)}$, after which good feeding practices are encouraged. During rehabilitation of children with SAM, emphasis is put on adequate intake of energy and protein, as well as on micronutrients, whereas PUFA intake is given less attention.

Fatty acids are good sources of energy. However, effects of $n-3$ and $n-6$ fatty acids go far beyond their role as fuels, as they are essential nutrients with important physiological functions. The $n$-6 PUFA linoleic acid (LA, $18: 2 n-6$ ) is incorporated in skin ceramides, whereas arachidonic acid (AA, 20:4n-6) in cell membranes acts as a precursor of eicosanoid ${ }^{(5)}$. A deficiency in these fatty acids can therefore result in scaly skin, reduced growth and increased infections among others. The main function of $n$-3 PUFA is exerted via DHA (22:6n-3) in central nervous system membranes, where they have a structural role, as well as a role in functional processes that are not completely clarified $^{(5)}$. A lack of essential $n-6$ and $n-3$ fatty acids will result in increased production of Mead acid (20:3n-9) and $n-6$ docosapentanoic acid (DPA) (22:5n-6), the metabolical counter parts of AA and DHA, respectively, that do not fulfil

Abbreviations: AA, arachidonic acid; ALA, $\alpha$-linolenic acid; DPA, docosapentanoic acid; \%E, percentage of energy intake; FA\%, fatty acid percentage; LA, linoleic acid; LCPUFA, long-chain PUFA; NG, nasogastric; RUTF, ready-to-use therapeutic feed; SAM, severe acute malnutrition.

* Corresponding author: E. Babirekere-Iriso, email ebabirekere@yahoo.com 
their essential functions. These two fatty acids may therefore indicate $n-3$ and $n-6$ deficiency, sometimes reported as ratios with their respective $n-6$ and $n-3$ fatty acid counterparts.

A number of studies show associations between SAM and a low $n-3$ and $n$ - 6 PUFA status ${ }^{(6)}$. However, limited data exist on the effect of recovery from SAM on LA, $\alpha$-linolenic acid (ALA, $18: 3 n-3)$ and long-chain (LC) PUFA status (LCPUFA defined as PUFA with $>18$ carbon atoms and $>3$ double bonds). A study of Nigerian hospitalised children with SAM found significant increases in LA values and decreases in AA values after 2 weeks of treatment with a therapeutic high-energy and high-protein diet with maize and milk, eggs, beans, vegetables, fish, meat, vegetable and palm oil ${ }^{(7)}$. ALA values did not change during hospitalisation, but the DHA content of plasma phospholipids increased significantly ${ }^{(7)}$. A recent study of Kenyan children with SAM found that $n-3$ and $n-6$ PUFA requirements of children with SAM were not met by the current formulation of RUTF or by a RUTF with elevated levels of ALA but without $n-3$ LCPUFA $^{(8)}$. Another study of Malawian children with SAM compared standard RUTF with a novel RUTF with relatively less LA and more oleic acid (HO-RUTF) to children and found positive changes in plasma phospholipid DHA and EPA $(20: 5 n-3)$ in the HO-RUTF group compared with the RUTF group $^{(9)}$. No studies have investigated the effects of F-75 and F-100 during in-hospital treatment of SAM on children's fatty acid status.

This prospective study investigates changes in whole-blood PUFA in Ugandan children with SAM from hospital admission to transition, discharge and follow-up after 8 and 16 weeks. We furthermore describe associations between changes in wholeblood PUFA values and clinical conditions during treatment.

\section{Methods}

\section{Study design, site and standard treatment}

This prospective observational study followed up children with in-hospital treatment of SAM between October 2012 and June 2013.

Mwanamugimu Nutrition Unit at Mulago Hospital is the main treatment centre for children with complicated SAM in Uganda. Children received standard in-patient treatment according to the Ugandan National Protocol for the Integrated Management of Acute Malnutrition ${ }^{(10)}$, based on recommendations from the World Health Organization ${ }^{(11)}$. Children were given therapeutic diets, F-75 and F-100 (Nutriset) and empirical parenteral antibiotics, usually ampicillin and gentamicin. Small, frequent feeds of F-75 were given at a rate of $100-130 \mathrm{ml} / \mathrm{kg}$ body weight per $\mathrm{d}$, either orally or by the nasogastric (NG) tube. After stabilisation, the children were changed from F-75 to catch-up milkbased formula $\mathrm{F}-100^{(2)}$. After about $2-3 \mathrm{~d}$ of transition, the volume of F-100 was gradually increased to a maximum of $220 \mathrm{ml} / \mathrm{kg}$ per $\mathrm{d}$. When the children were clinically well, had regained appetite and had no oedema, they were discharged for out-patient treatment with RUTF. The children were then followed up in the hospital's out-patient clinic every 2 weeks until recovery from SAM, as indicated by $20 \%$ weight gain, no oedema for two consecutive follow-up visits and increasing mid-upper arm circumference (MUAC) according to recommendations at the time of study ${ }^{(12)}$. Biological mothers were offered routine counselling and testing for HIV antibodies according to WHO guidelines, using Determine ${ }^{\mathrm{TM}}$ rapid test as first-line ${ }^{(13)}$. If the mother was infected or absent, the child was tested.

A few local modifications of the protocol were practiced at the time of the study: if a child had diarrhoea and milk intolerance was suspected, the diet was fully or partially replaced by rice porridge for some days. Furthermore, most children were given a daily serving of maize-soya-porridge during and after the transition phase, and these maize-soyabased products' composition varied. In preparation for discharge, all mothers participated in cooking classes preparing energy-dense complementary foods using locally available ingredients, which was also served to the children.

\section{Selection criteria}

Children 6-59 months of age with SAM, defined as weightfor-height $z$-score (WHZ) $<-3$ of the WHO Growth Standard, MUAC $<11.5 \mathrm{~cm}$ or bilateral pitting oedema, and who lived near the hospital were eligible for the study. Those who were in shock at admission, had severe respiratory distress requiring resuscitation, $\mathrm{Hb}$ concentration $<4 \mathrm{~g} / \mathrm{dl}$, body weight $<4.5 \mathrm{~kg}$ or significant disability such as cerebral palsy were excluded. Inclusion was not possible during weekends or public holidays.

A control group of healthy well-nourished children was recruited among siblings of the admitted children and children of hospital staff. The inclusion criteria for these children were being apparently healthy, aged 6-59 months old and having a WHZ $>-1$.

\section{Sample size}

The sample size of 120 was based on the main outcome, plasma phosphate $^{(14)}$. With 120 SAM children, we would have $80 \%$ power to detect a difference of $0.5 \mathrm{SD}$ or more in any normally distributed variable between two groups of equal size, with a $5 \%$ significance level. Similarly, with a control group of thirty children, we would have $80 \%$ power to detect differences of 0.6 SD or more between SAM and control.

\section{Ethical issues}

Approval of the study was obtained from the Makerere University School of Medicine Research Ethics Committee, and Uganda National Council of Science and Technology, and a consultative approval was obtained from the Danish National Board of Research Ethics. Informed consent was provided by parents or guardians of the children before enrolment into the study. Regardless of participation in the study, all children admitted with SAM received similar routine medical and nutritional treatment.

\section{Data collection}

A questionnaire was used to obtain socio-demographic information, as well as feeding and medical history. Physical 
examination including vital signs and checking of bilateral pitting oedema was performed. Anthropometric measurements involved taking MUAC using a tape (MUAC Child Red/Pac-50) and measurement of length to the nearest $1 \mathrm{~mm}$ using an infant length board (Infant/Child ShorrBoard ${ }^{\circledR}$ ). Body weight was measured daily to the nearest $100 \mathrm{~g}$ using a digital scale (Seca 813; Seca GmbH \& Co.). Anthropometric $z$-scores were computed using WHO Growth Standards ${ }^{(15)}$ adjusting for the fact that length was measured even in children $<2$ years, and using the lowest weight recorded during admission, to determine weight free from oedema.

\section{Blood sampling}

On admission, $\mathrm{Hb}$ was measured in venous blood collected in heparinized vacutainer tubes (Becton Dickinson) using a $\mathrm{Hb}$ 201+ (HemoCue). At admission, transition and discharge, approximately $40 \mu \mathrm{l}$ of heparinized whole blood was applied to a chromatography paper strip (grade 3MM; Whatman), from which potential lipid contaminants had previously been removed with chloroform and methanol, and treated with antioxidants $(1000 \mu \mathrm{g}$ deferoxamine and $50 \mu \mathrm{g}$ butylated hydroxy toluene) ${ }^{(16)}$ so that approximately $1 \mathrm{~cm}^{2}$ of the strip was saturated with blood. At 8 and 16 weeks of follow-up, blood samples were obtained by finger-prick and applied to antioxidant-treated chromatography paper strip. Results from analysis of dried blood spots collected from fingertips have been compared with venous blood samples and found to give substantially identical information ${ }^{(17)}$ The blood spots were allowed to dry completely at room temperature and were then stored in a sealed container (polypropylene 'ziplock' bag) in a refrigerator for up to 2 months ${ }^{(18)}$ until they were shipped to University of Waterloo, Department of Kinesiology, Canada, for fatty acid analysis.

Whole-blood spots were chosen as the method of sample preparation because of the simplified method for blood collection, storage and shipment to the analytical laboratory where samples are easily processed for analysis ${ }^{(17)}$. Furthermore, whole-blood fatty acid analysis includes all lipid fractions and is most representative of the body fatty acid status, when compared with fatty acids in plasma, blood cells such as erythrocytes and platelets ${ }^{(19,20)}$.

Plasma was obtained from a vacutainer with citrate (CellPreparation Tube; Becton Dickinson) by centrifugation at $1300-2200 \mathrm{~g}$ for $20 \mathrm{~min}$, and it was stored at $-80^{\circ} \mathrm{C}$ until shipped on dry ice to the University of Copenhagen, Department of Nutrition, Exercise and Sports, Denmark, where plasma level of C-reactive protein (CRP) was measured using the highsensitivity kit on a ABX Pentra 400 (Horiba, no. A11A01611 and A11A01696).

\section{Fatty acid analysis}

Fatty acid methyl esters were prepared from the whole-blood spots by direct trans-esterification, and they were analysed by high-throughput $\mathrm{GLC}^{(21,22)}$. Briefly, the whole-blood spot was directly trans-esterified with the addition of $1 \mathrm{ml}$ of $14 \%$ boron trifluoride in methanol (Pierce Chemicals), $300 \mu \mathrm{l}$ of hexane and
$3 \mu \mathrm{g}$ of an internal standard ( $22: 3 n-3$ ethyl ester; Nu-Chek Prep) and heating at $95^{\circ} \mathrm{C}$ for $1 \mathrm{~h}^{(22)}$. Samples were allowed to cool to room temperature, after which water and hexane were added $(1 \mathrm{ml}$ each). The samples were vortexed for $1 \mathrm{~min}$, and then centrifuged for $5 \mathrm{~min}$ at $3000 \mathrm{rpm}$ to separate the organic and aqueous phases. The organic upper hexane layer containing fatty acid methyl esters was collected, dried under a stream of $\mathrm{N}$ and reconstituted in hexane. Fatty acid methyl esters were analysed on a Varian 3900 gas chromatograph equipped with a capillary column of $15 \mathrm{~m} \times 0 \cdot 10 \mathrm{~mm}$ i.d. $\times 0 \cdot 10 \mu \mathrm{m}$ thick film of nitroterephthalic acid-modified polyethylene glycol (DB-FFAP from $\mathrm{J} \& \mathrm{~W}$ Scientific, Agilent Technologies) and hydrogen as the carrier gas ${ }^{(22)}$. Samples $(1 \mu \mathrm{l})$ were introduced by a Varian CP-8400 autosampler into the $250^{\circ} \mathrm{C}$ injector with a split ratio of 100:1. The initial temperature was $150^{\circ} \mathrm{C}$, which was held for $0.25 \mathrm{~min}$, followed by a $35^{\circ} \mathrm{C} / \mathrm{min}$ ramp to $200^{\circ} \mathrm{C}$, an $8^{\circ} \mathrm{C} / \mathrm{min}$ ramp to $225^{\circ} \mathrm{C}$, where it was kept for $3.2 \mathrm{~min}$, and then an $80^{\circ} \mathrm{C} / \mathrm{min}$ ramp up to $245^{\circ} \mathrm{C}$, with a 15 -min hold at the end ${ }^{(22)}$. The flame ionisation detector temperature was $300^{\circ} \mathrm{C}$, with air and $\mathrm{N}$ make-up gas flow rates of 300 and $25 \mathrm{ml} / \mathrm{min}$, respectively, and a sampling frequency of $80 \mathrm{~Hz}$. Peaks were identified (thirty-four fatty acids) by comparing retention times with an external mixed standard sample (GLC-462, Nu-Chek Prep Inc.). Concentrations of individual fatty acids were determined by comparing peak areas to the response of the known concentration of the internal standard. Individual fatty acids were also expressed as the weight percentage of total fatty acids (FA\%). Unknown peaks were included in the calculations for weight percentage, and for each sample the identified fatty acids accounted for over $95 \%$ of the total fatty acid concentration. Various sums and ratios of fatty acids were calculated from the weight percentage fatty acid data.

\section{Statistics}

Double data entry was done into Epidata, and analysis was performed using Stata 12 (StataCorp LP). $t$ Test and $\chi^{2}$ tests were used to test for differences in means and proportions between children with SAM and controls. Paired $t$ test was used to analyse change in fatty acid values at different times during follow-up. ANCOVA in the changes of mean values from admission was done to identify predictors of changes in PUFA with adjustment for age and sex. $P$-values $<0.05$ were considered statistically significant.

\section{Results}

The study recruited 120 children with a median age of 15.9 months. In all, $38 \%$ were female, $64 \%$ had oedema and $19 \%$ were HIV infected (Table 1). Control children and the children with SAM were similar in age and sex. Other data from the same children have been published previously ${ }^{(23,24)}$.

The median duration of hospital admission was $19 \mathrm{~d}$. At admission, blood samples were obtained from 108 of the 120 recruited children with SAM. Before transition, eight children died, five self-discharged and one was excluded because of very low blood $\mathrm{Hb}$, leaving 106 children going through 
Table 1. Baseline characteristics of children with severe acute malnutrition (SAM) and healthy controls* (Medians and 25, 75 percentiles; numbers and percentages; mean values and standard deviations)

\begin{tabular}{|c|c|c|c|c|c|}
\hline & \multicolumn{2}{|c|}{ Children with SAM ( $n$ 120) } & \multicolumn{2}{|c|}{ Control children ( $n$ 29) } & \multirow[b]{2}{*}{$P$} \\
\hline & $n$ & $\%$ & $n$ & $\%$ & \\
\hline Age (months) & & & & & 0.194 \\
\hline Median & \multicolumn{2}{|c|}{$15 \cdot 9$} & \multicolumn{2}{|c|}{$16 \cdot 3$} & \\
\hline 25; 75 percentiles & \multicolumn{2}{|c|}{$12 \cdot 6 ; 21 \cdot 9$} & \multicolumn{2}{|c|}{$11 \cdot 3 ; 24 \cdot 6$} & \\
\hline Sex & & & & & 0.521 \\
\hline Male & 74 & 62 & 16 & 55 & \\
\hline Female & 46 & 38 & 13 & 45 & \\
\hline Weight-for-height $z$-score $\dagger$ & -3.4 & 1.4 & 0.8 & 0.9 & $<0.001$ \\
\hline Weight gain at discharge $(\mathrm{g} / \mathrm{kg}$ per $\mathrm{d}) \dagger$ & $5 \cdot 8$ & $3 \cdot 2$ & N/A & & \\
\hline Weight gain at 8 of weeks follow-up ( $\mathrm{g} / \mathrm{kg}$ per $\mathrm{d}) \dagger$ & 4.9 & $2 \cdot 3$ & $\mathrm{~N} / \mathrm{A}$ & & \\
\hline Height-for-age $z$-score $\dagger$ & $-3 \cdot 1$ & 1.5 & -0.9 & 1.1 & $<0.001$ \\
\hline Height gain at discharge $(\mathrm{cm}) \dagger$ & -0.2 & 1.6 & $\mathrm{~N} / \mathrm{A}$ & & \\
\hline Height gain at 8 weeks of follow-up $(\mathrm{cm}) \dagger$ & 0.8 & 1.4 & N/A & & \\
\hline Mother's education & & & & & 0.410 \\
\hline No school & 6 & 6 & 1 & 4 & \\
\hline Primary school & 42 & 43 & 10 & 35 & \\
\hline Secondary school & 42 & 43 & 14 & 48 & \\
\hline More than secondary school & 7 & 7 & 3 & 10 & \\
\hline Mother's BMI $\left(\mathrm{kg} / \mathrm{m}^{2}\right) \dagger$ & $21 \cdot 7$ & $2 \cdot 7$ & $22 \cdot 0$ & $4 \cdot 3$ & 0.753 \\
\hline Breast-feeding & & & & & $<0.001$ \\
\hline Breast-feeding & 19 & 17 & 15 & 52 & \\
\hline Not breast-feeding & 92 & 83 & 14 & 48 & \\
\hline Fish served in household in last 2 weeks & & & & & 0.072 \\
\hline Yes & 62 & 62 & 21 & 81 & \\
\hline No & 38 & 38 & 5 & 19 & \\
\hline $\mathrm{Hb}(\mathrm{g} / \mathrm{dl}) \dagger$ & 9.0 & $2 \cdot 3$ & $10 \cdot 2$ & 1.5 & 0.015 \\
\hline Serum CRP (mg/l) & & & & & 0.006 \\
\hline Median & & & & & \\
\hline 25; 75 percentiles & & & & & \\
\hline Oedema & & & $\mathrm{N} / \mathrm{A}$ & & \\
\hline Present & 77 & 64 & & & \\
\hline Absent & 43 & 36 & & & \\
\hline HIV status & & & - & & \\
\hline Infected & 20 & 19 & & & \\
\hline Not infected & 84 & 81 & & & \\
\hline
\end{tabular}

CRP, C-reactive protein; -, data not available.

${ }^{*}$ Numbers in categories may not sum up because of missing data. Data on rate of weight gain at discharge were available for eighty-two SAM children and on weight gain at 8 weeks of follow-up on sixty-six children. Data on height gain at discharge and follow-up were available on eighty-three and sixty-five SAM children, respectively. Data on mothers BMI were available for eighty-one SAM and seven control children. Similarly, data were available on Hb from 112 SAM and twenty-five control children, on CRP from eighty-three SAM and twenty control children and on HIV from 104 SAM children. $t$ Test or $X^{2}$ tests were used to compare children with SAM and healthy controls.

†Mean and standard deviation.

transition, and blood samples were obtained from seventy-six children. From transition to hospital discharge, nine children died and thirteen self-discharged or withdrew their consent to participate. Accordingly, eighty-three children were discharged, and blood samples were obtained from seventy-two children. At first follow-up 8 weeks after admission, sixty-six children turned up, and blood samples were obtained from forty-three children, whereas forty-nine children came for follow-up at 16 weeks after admission and blood samples were obtained from forty-four children.

The children with SAM presented with lower LCPUFA values at admission compared with healthy controls (Table 2). The mean values of most SFA were lower in children with SAM until 8 weeks of follow-up (for total SFA, $44.7 \mathrm{FA} \%$ at transition and $44.4 \mathrm{FA} \%$ at discharge $v .47 .7 \mathrm{FA} \%$ for the control children, both $P<0.001)$. The proportion of most MUFA remained higher in children with SAM throughout the follow-up period (for total MUFA, 31.0 FA\% at admission, 33.5 FA\% at transition, $30.7 \mathrm{FA} \%$ at discharge, $27 \cdot 2 \mathrm{FA} \%$ at 8 weeks and $27 \cdot 8 \mathrm{FA} \%$ at 16 weeks follow-up $v .24 .5 \mathrm{FA} \%$ for the controls, $P<0.001$ for all time periods except 8 weeks of follow-up, which was 0.001). The total values of $n-6$ PUFA in children with SAM increased to values similar to the controls at 8 weeks of follow-up, but it again decreased at 16 weeks of follow-up (20.0 FA\% for SAM children $v .23 .1 \mathrm{FA} \%$ for controls, $P<0 \cdot 001)$. At discharge, all individual $n$-6 PUFA became similar to values in the control children, except AA, which remained lower (4.42 FA\% for SAM children compared with 5.95 FA\% in controls, $P<0.001)$. $n-6$ LCPUFA were similar to the controls by 8 weeks of follow-up. Individual and total $n$-3 PUFA values decreased after admission, apart from ALA, which was similar to healthy children throughout follow-up. The ratio of $n-6: n-3$ PUFA was lower in children with SAM than in the healthy controls at admission (7.9 v. 8.9, $P=0.043$ ), but it became higher in children with SAM at all times after initiation of the treatment. The Mead acid:AA ratio remained higher in children with SAM throughout the entire period of treatment compared with the control children. 
Table 2. Fatty acid values during treatment of children with severe acute malnutrition (SAM) and healthy controls $†$ (Mean values and standard deviations)

\begin{tabular}{|c|c|c|c|c|c|c|c|c|c|c|c|c|}
\hline \multirow[b]{3}{*}{ Fatty acid } & \multicolumn{10}{|c|}{ Children with SAM } & & \\
\hline & \multicolumn{2}{|c|}{ Admission ( $n$ 108) } & \multicolumn{2}{|c|}{ Transition $(n 76)$} & \multicolumn{2}{|c|}{ Discharge $(n$ 72) } & \multicolumn{2}{|c|}{8 weeks $(n 43)$} & \multicolumn{2}{|c|}{16 weeks $(n 44)$} & \multicolumn{2}{|c|}{ Control children $(n$ 24) } \\
\hline & Mean & SD & Mean & SD & Mean & SD & Mean & SD & Mean & SD & Mean & SD \\
\hline \multicolumn{13}{|l|}{ Saturated } \\
\hline $12: 0$ & $0 \cdot 20^{*}$ & 0.20 & 0.24 & 0.26 & $0.19^{\star}$ & 0.23 & 0.23 & 0.21 & $0.22^{*}$ & 0.19 & 0.37 & 0.41 \\
\hline $14: 0$ & $1.41^{*}$ & 0.60 & $1 \cdot 20^{* *}$ & 0.51 & $1.09^{* *}$ & 0.67 & 1.42 & 0.80 & $1.33^{*}$ & 0.67 & 1.84 & 1.01 \\
\hline $16: 0$ & $28 \cdot 8^{\star}$ & $1 \cdot 8$ & $28 \cdot 9^{\star \star}$ & 1.9 & 27.5 & 1.9 & $27 \cdot 1$ & 1.6 & $27 \cdot 7$ & $1 \cdot 8$ & $27 \cdot 4$ & 1.5 \\
\hline $17: 0$ & $0.34^{\star *}$ & 0.09 & $0.25^{\star *}$ & 0.07 & $0.23^{* *}$ & 0.06 & 0.53 & 1.18 & 0.39 & 0.12 & 0.41 & 0.10 \\
\hline $18: 0$ & $13 \cdot 0$ & $2 \cdot 3$ & $11 \cdot 4^{\star \star}$ & $2 \cdot 1$ & $12 \cdot 0^{*}$ & $2 \cdot 1$ & $13 \cdot 6$ & $2 \cdot 8$ & $13 \cdot 6$ & 2.9 & 13.5 & 1.3 \\
\hline $20: 0$ & $0.27^{\star \star}$ & 0.06 & $0.29^{\star \star}$ & 0.07 & $0.31^{*}$ & 0.05 & $0.38^{\star}$ & 0.09 & 0.36 & 0.07 & 0.34 & 0.06 \\
\hline $22: 0$ & $0.59^{* *}$ & 0.16 & $0.55^{\star *}$ & 0.17 & $0.66^{\star *}$ & 0.15 & $0.97^{\star}$ & 0.23 & 0.89 & 0.24 & 0.86 & 0.17 \\
\hline $23: 0$ & $0 \cdot 10^{\star *}$ & 0.05 & $0.09^{* \star}$ & 0.04 & $0.11^{* *}$ & 0.03 & $0.15^{\star}$ & 0.04 & 0.15 & 0.05 & 0.17 & 0.05 \\
\hline $24: 0$ & $0.94^{\star *}$ & 0.33 & $0.83^{* *}$ & 0.30 & $1.02^{* *}$ & 0.39 & 1.50 & 0.37 & 1.49 & 0.41 & 1.44 & 0.33 \\
\hline SFA & $46 \cdot 7$ & $2 \cdot 6$ & $44 \cdot 7^{\star \star}$ & 3.0 & $44 \cdot 4^{\star \star}$ & 3.3 & $46 \cdot 8$ & $4 \cdot 1$ & $47 \cdot 2$ & 3.8 & $47 \cdot 7$ & 1.6 \\
\hline \multicolumn{13}{|l|}{ Unsaturated } \\
\hline $16: 1 n-7$ & 1.41 & 0.56 & 1.68 & 0.86 & 1.21 & 0.57 & 1.28 & 0.71 & 1.75 & 0.76 & 1.41 & 0.56 \\
\hline $18: 1 n-7$ & $2 \cdot 10^{\star *}$ & 0.36 & 1.70 & 0.38 & 1.57 & 0.42 & $1.49^{*}$ & 0.27 & 1.59 & 0.29 & 1.70 & 0.36 \\
\hline $18: 1 n-9$ & $23 \cdot 9^{* \star}$ & $3 \cdot 1$ & $27 \cdot 9^{*}$ & $3 \cdot 8$ & $25 \cdot 3^{* *}$ & 3.2 & $21 \cdot 6^{\star}$ & 3.0 & $21 \cdot 8^{\star \star}$ & $2 \cdot 9$ & $19 \cdot 0$ & 2.5 \\
\hline $20: 1 n-9$ & $0.25^{\star}$ & 0.44 & $0.24^{*}$ & 0.04 & 0.30 & 0.28 & $0.32^{\star \star}$ & 0.05 & $0.27^{\star \star}$ & 0.06 & 0.21 & 0.45 \\
\hline $22: 1 n-9$ & $0.96^{*}$ & 0.60 & 0.78 & 0.54 & 0.83 & 0.99 & $0.95^{\star *}$ & 0.36 & $0.87^{\star}$ & 0.48 & 0.64 & 0.18 \\
\hline $24: 1 n-9$ & 1.19 & 0.34 & $1.06^{*}$ & 0.32 & 1.30 & 0.40 & 1.39 & 0.30 & 1.31 & 0.30 & 1.29 & 0.27 \\
\hline MUFA & $31 \cdot 0^{\star *}$ & $3 \cdot 3$ & $33 \cdot 5^{\star \star}$ & $3 \cdot 7$ & $30 \cdot 7^{\star \star}$ & 3.7 & $27 \cdot 2^{\star}$ & $3 \cdot 1$ & $27 \cdot 8^{\star \star}$ & 3.0 & 24.5 & $2 \cdot 8$ \\
\hline Mead acid $(20: 3 n-9)$ & 0.14 & $0 \cdot 10$ & 0.15 & 0.15 & $0.19^{*}$ & 0.13 & $0.13^{*}$ & 0.06 & $0.15^{\star}$ & 0.07 & 0.11 & 0.06 \\
\hline \multicolumn{13}{|l|}{$n-6$ PUFA } \\
\hline LA $(18: 2 n-6)$ & $10 \cdot 4^{\star *}$ & 2.5 & $12 \cdot 1^{\star \star}$ & $2 \cdot 0$ & 13.7 & 2.5 & 13.9 & 3.0 & $12 \cdot 4^{\star \star}$ & $2 \cdot 2$ & $14 \cdot 8$ & $2 \cdot 2$ \\
\hline $18: 3 n-6$ & $0.07^{\star \star}$ & 0.04 & 0.11 & 0.06 & 0.12 & 0.06 & 0.12 & 0.05 & $0.16^{\star}$ & 0.08 & 0.12 & 0.07 \\
\hline $20: 2 n-6$ & $0.16^{*}$ & 0.04 & $0.13^{\star \star}$ & 0.04 & 0.17 & 0.04 & $0.22^{*}$ & 0.06 & 0.18 & 0.04 & 0.18 & 0.04 \\
\hline $20: 3 n-6$ & $0.57^{\star *}$ & 0.13 & $0.58^{\star *}$ & 0.14 & 0.85 & 0.23 & 0.93 & 0.19 & 0.88 & 0.20 & 0.90 & 0.18 \\
\hline AA $(20: 4 n-6)$ & $4 \cdot 67^{\star \star}$ & 1.07 & $3.54^{\star \star}$ & 0.90 & $4.42^{\star \star}$ & 1.07 & $5 \cdot 09^{*}$ & 1.17 & $5 \cdot 07^{*}$ & 1.47 & 5.95 & 1.01 \\
\hline $22: 4 n-6$ & $0.62^{*}$ & 0.20 & $0.54^{\star *}$ & $0 \cdot 17$ & 0.72 & 0.23 & 0.90 & 0.27 & 0.84 & 0.34 & 0.77 & 0.33 \\
\hline $22: 5 n-6$ & $0.26^{*}$ & 0.08 & $0.21^{\star \star}$ & 0.06 & 0.28 & 0.09 & 0.32 & 0.09 & 0.31 & 0.11 & 0.31 & 0.09 \\
\hline$n-6$ LCPUFA & $6 \cdot 2^{\star \star}$ & $1 \cdot 3$ & $4 \cdot 9^{\star \star}$ & $1 \cdot 1$ & $6 \cdot 3^{\star \star}$ & 1.4 & $7 \cdot 3$ & 1.6 & $7 \cdot 2$ & $2 \cdot 0$ & 8.0 & 1.4 \\
\hline$n-6$ Fatty acids & $16 \cdot 8^{\star \star}$ & $2 \cdot 7$ & $17 \cdot 3^{\star \star}$ & 2.5 & $20 \cdot 3^{* *}$ & 3.0 & 21.5 & 3.9 & $20 \cdot 0^{\star *}$ & $2 \cdot 6$ & $23 \cdot 1$ & 3.0 \\
\hline \multicolumn{13}{|l|}{$n-3$ PUFA } \\
\hline ALA $(18: 3 n-3)$ & 0.17 & 0.07 & $0.14^{*}$ & 0.05 & 0.17 & 0.11 & 0.16 & 0.07 & 0.20 & 0.11 & 0.19 & 0.11 \\
\hline EPA $(20: 5 n-3)$ & 0.18 & 0.08 & $0.11^{\star \star}$ & 0.05 & $0.12^{\star \star}$ & 0.05 & $0.12^{\star \star}$ & 0.05 & $0.14^{\star \star}$ & 0.06 & 0.20 & 0.09 \\
\hline $22: 5 n-3$ & 0.49 & 0.16 & $0.37^{\star \star}$ & 0.13 & $0.41^{*}$ & 0.14 & $0.41^{\star}$ & 0.10 & $0.43^{\star}$ & 0.15 & 0.51 & 0.11 \\
\hline DHA $(22: 6 n-3)$ & $1.40^{\star *}$ & 0.45 & $1.08^{\star \star}$ & 0.42 & $1 \cdot 22^{\star *}$ & 0.51 & $1.06^{\star \star}$ & 0.46 & $1 \cdot 14^{\star *}$ & 0.56 & 1.78 & 0.54 \\
\hline$n-3$ LCPUFA & $2 \cdot 10^{*}$ & 0.57 & $1.59^{\star *}$ & 0.52 & $1.77^{\star *}$ & 0.64 & $1.64^{\star *}$ & 0.52 & $1.74^{\star \star}$ & 0.71 & 2.53 & 0.65 \\
\hline$n-3$ Fatty acids & $2 \cdot 26^{*}$ & 0.58 & $1.73^{\star \star}$ & 0.54 & $1.94^{* *}$ & 0.68 & $1.80^{* *}$ & 0.54 & $1.94^{\star *}$ & 0.71 & 2.72 & 0.65 \\
\hline PUFA & $19 \cdot 0^{\star \star}$ & 2.9 & $19 \cdot 0^{\star \star}$ & $2 \cdot 8$ & $22 \cdot 2^{\star \star}$ & 3.5 & $23 \cdot 3^{*}$ & $4 \cdot 1$ & $21 \cdot 9^{\star \star}$ & $2 \cdot 9$ & $25 \cdot 8$ & $3 \cdot 2$ \\
\hline Total fatty acid concentration & 277 & 86 & $326^{*}$ & 118 & $307^{\star}$ & 100 & 238 & 86 & 238 & 66 & 246 & 52 \\
\hline$n-6: n-3$ PUFA ratio & $7 \cdot 9^{*}$ & 2.3 & $10 \cdot 8^{*}$ & 2.9 & $11.4^{\star}$ & 3.3 & $12 \cdot 9^{* \star}$ & 4.1 & $11 \cdot 6^{*}$ & 4.3 & 8.9 & $2 \cdot 2$ \\
\hline Mead:AA ratio & $0.03^{*}$ & 0.02 & $0.05^{*}$ & 0.06 & $0.05^{\star *}$ & 0.03 & $0.03^{*}$ & 0.01 & $0.03^{*}$ & 0.01 & 0.02 & 0.01 \\
\hline EPA:AA ratio & 0.04 & 0.02 & 0.03 & 0.01 & $0.03^{*}$ & 0.01 & $0.03^{*}$ & 0.01 & $0.03^{*}$ & 0.01 & 0.04 & 0.02 \\
\hline $22: 5 n-6:$ DHA ratio & 0.20 & 0.08 & 0.22 & 0.08 & $0.26^{*}$ & 0.10 & $0.34^{* *}$ & 0.14 & $0.31^{\star *}$ & 0.13 & 0.19 & 0.08 \\
\hline
\end{tabular}

$n$, Number of children in whom information was available; LA, linoleic acid; AA, arachidonic acid; LCPUFA, long-chain PUFA; $n-6: n-3$ PUFA ratio, ratio of total $n-6$ PUFA:total $n$-3 PUFA.

${ }^{*} P<0.05$ and ${ }^{* \star} P<0.001$ relative to control children. Paired $t$ test analyses were done to test for differences in fatty acids between children with SAM at different times of follow-up and healthy controls.

† Data for individual fatty acids and fatty acid classes are given as weight percentage of total fatty acids (FA\%) and total whole-blood fatty acid concentration is expressed as $\mu \mathrm{g} / 100 \mu \mathrm{l}$.

The mean changes in fatty acid values from admission to transition, discharge and follow-up are shown in Table 3. The mean of total SFA values decreased by 1.4 (95\% CI $0 \cdot 16,2 \cdot 19)$ FA\% during transition $(P=0 \cdot 001)$ and by $1 \cdot 6(95 \%$ CI $0 \cdot 18,2 \cdot 41)$ FA\% at discharge $(P<0 \cdot 001)$. This decrease was initially matched by an increase in the mean of total MUFA by 1.53 (95\% CI $0.43,2.63) \mathrm{FA} \%$ at transition $(P=0.007)$ but followed by a decrease at 8 and 16 weeks of follow-up (3.6; $95 \%$ CI 2.39, $4.88 \mathrm{FA} \%$ and $3 \cdot 2 ; 95 \%$ CI $2 \cdot 0,4 \cdot 47 \mathrm{FA} \%$, respectively, $P<0.001)$. Generally, values of $n-6$ PUFA increased by 16 weeks of follow-up, whereas $n-3$ PUFA decreased.
Fig. 1 shows changes in values of PUFA classes and selected PUFA during treatment and follow-up. LA values increased initially at transition and discharge, remained high at 8 weeks of follow-up and then decreased at 16 weeks. AA values reached a nadir at transition, followed by a small increase at discharge through to 16 weeks of follow-up. The percentage contribution of total $n-3$ PUFA dropped already at transition and never returned to admission values.

Predictors of changes in fatty acid values of children with SAM from admission to discharge adjusted for age and sex are summarised in Table 4. Weight gain during hospital treatment 
Table 3. Mean changes in fatty acid values during treatment in children with severe acute malnutrition $\dagger$ (Regression coefficients $(b)$ and $95 \%$ confidence intervals)

\begin{tabular}{|c|c|c|c|c|c|c|c|c|}
\hline \multirow[b]{2}{*}{ Fatty acid } & \multicolumn{2}{|c|}{ Transition ( $n$ 76) } & \multicolumn{2}{|c|}{ Discharge ( $n 72)$} & \multicolumn{2}{|c|}{8 weeks ( $n$ 43) } & \multicolumn{2}{|c|}{16 weeks ( $n$ 44) } \\
\hline & $b$ & $95 \% \mathrm{Cl}$ & $b$ & $95 \% \mathrm{Cl}$ & $b$ & $95 \% \mathrm{Cl}$ & $b$ & $95 \% \mathrm{Cl}$ \\
\hline \multicolumn{9}{|l|}{ Saturated } \\
\hline $12: 0$ & 0.04 & $-0.04,0.11$ & 0.01 & $-0.07,0.08$ & 0.05 & $-0.04,0.13$ & 0.04 & $-0.04,0.13$ \\
\hline $14: 0$ & -0.14 & $-0.34,0.07$ & -0.21 & $-0.42,-0.01^{*}$ & 0.08 & $-0.15,0.31$ & 0.02 & $-0.21,0.25$ \\
\hline $16: 0$ & 0.00 & $-0.47,0.47$ & -1.07 & $-1.54,-0.60^{\star \star}$ & -1.35 & $-1.88,-0.83^{\star \star}$ & -0.93 & $-1.46,-0.41^{\star}$ \\
\hline $17: 0$ & -0.06 & $-0.16,0.04$ & -0.06 & $-0.16,0.04$ & 0.14 & $0.03,0.25^{\star}$ & 0.05 & $-0.06,0.16$ \\
\hline $18: 0$ & -1.14 & $-1.71,-0.57^{\star *}$ & -0.68 & $-1 \cdot 26,-0 \cdot 11^{*}$ & 0.51 & $-0.14,1.15$ & 0.49 & $-0.15,1.13$ \\
\hline $20: 0$ & 0.01 & $-0.00,0.03$ & 0.03 & $0.02,0.05^{\star \star}$ & 0.08 & $0.06,0.10^{\star \star}$ & 0.07 & $0.05,0.09^{\star \star}$ \\
\hline $22: 0$ & -0.01 & $-0.06,0.04$ & 0.07 & $0.02,0.12^{\star}$ & 0.29 & $0.23,0.35^{\star \star}$ & 0.24 & $0.18,0.30^{\star *}$ \\
\hline $23: 0$ & -0.00 & $-0.02,0.01$ & 0.01 & $-0.00,0.02$ & 0.04 & $0.03,0.06^{\star *}$ & 0.05 & $0.03,0.06^{* *}$ \\
\hline $24: 0$ & -0.06 & $-0.16,0.05$ & 0.09 & $-0.01,0.19$ & 0.44 & $0.33,0.56^{\star *}$ & 0.44 & $0.32,0.55^{\star *}$ \\
\hline SFA & -1.40 & $-2 \cdot 19,-0.61^{*}$ & -1.61 & $-2.41,-0.81^{\star \star}$ & 0.27 & $-0.62,1.17$ & 0.50 & $-0.40,1.39$ \\
\hline \multicolumn{9}{|l|}{ Unsaturated } \\
\hline $16: 1 n-7$ & -0.64 & $-0.83,-0.44^{\star *}$ & -0.99 & $-1 \cdot 19,-0.79^{\star *}$ & -0.92 & $-1 \cdot 14,-0 \cdot 70^{\star *}$ & -0.62 & $-0.84,-0.40^{\star *}$ \\
\hline $18: 1 n-7$ & -0.33 & $-0.43,-0.24^{\star \star}$ & -0.43 & $-0.52,-0.33^{\star \star}$ & -0.46 & $-0.57,-0.36^{\star \star}$ & -0.40 & $-0.51,-0.29^{\star *}$ \\
\hline $18: 1 n-9$ & 2.77 & $1 \cdot 74,3 \cdot 79^{\star \star}$ & 0.68 & $-0.36,1.71$ & -2.37 & $-3 \cdot 53,-1 \cdot 21^{\star \star}$ & $-2 \cdot 20$ & $-3.36,-1.05^{\star *}$ \\
\hline $20: 1 n-9$ & -0.00 & $-0.04,0.03$ & 0.04 & $0.01,0.07^{\star}$ & 0.04 & $0.00,0.08^{\star}$ & 0.01 & $-0.02,0.05$ \\
\hline $22: 1 n-9$ & -0.16 & $-0.31,-0.01^{*}$ & -0.12 & $-0.27,0.03$ & -0.06 & $-0.23,0.11$ & -0.12 & $-0.29,0.06$ \\
\hline $24: 1 n-9$ & -0.09 & $-0.18,-0.01^{*}$ & 0.09 & $-0.00,0.17$ & 0.14 & $0.05,0.24^{\star}$ & 0.09 & $-0.00,0.19$ \\
\hline MUFA & 1.53 & $0.43,2 \cdot 63^{\star}$ & -0.72 & $-1.84,0.39$ & -3.63 & $-4 \cdot 88,-2 \cdot 39^{\star \star}$ & $-3 \cdot 23$ & $-4 \cdot 47,-2 \cdot 00^{\star \star}$ \\
\hline Mead acid $(20: 3 n-9)$ & 0.01 & $-0.02,0.03$ & 0.03 & $0.01,0.06^{\star}$ & -0.01 & $-0.04,0.03$ & -0.00 & $-0.03,0.03$ \\
\hline \multicolumn{9}{|l|}{$n-6$ PUFA } \\
\hline LA $(18: 2 n-6)$ & 1.59 & $0 \cdot 91,2 \cdot 28^{\star *}$ & $2 \cdot 77$ & $2.07,3.46^{\star \star}$ & 3.01 & $2 \cdot 23,3 \cdot 78^{\star *}$ & $2 \cdot 10$ & $1 \cdot 33,2 \cdot 87^{\star \star}$ \\
\hline $18: 3 n-6$ & 0.04 & $0.02,0.05^{\star \star}$ & 0.04 & $0.02,0.05^{\star \star}$ & 0.04 & $0.03,0.06^{\star \star}$ & 0.07 & $0.05,0.08^{\star *}$ \\
\hline $20: 2 n-6$ & -0.02 & $-0.03,-0.01^{\star}$ & 0.01 & $-0.00,0.02$ & 0.04 & $0.03,0.06^{\star \star}$ & 0.02 & $0.01,0.04^{\star}$ \\
\hline $20: 3 n-6$ & 0.03 & $-0.02,0.08$ & 0.23 & $0.18,0.29^{\star *}$ & 0.29 & $0.23,0.35^{\star \star}$ & 0.26 & $0.20,0.32^{\star \star}$ \\
\hline AA $(20: 4 n-6)$ & -0.79 & $-1.12,-0.46^{\star \star}$ & -0.10 & $-0.44,0.23$ & 0.50 & $0.12,0.87^{\star}$ & 0.48 & $0.11,0.85^{\star}$ \\
\hline $22: 4 n-6$ & -0.06 & $-0.13,0.01$ & 0.08 & $0.01,0.15^{\star}$ & 0.21 & $0.13,0.28^{\star *}$ & $0 \cdot 16$ & $0.09,0.24^{\star *}$ \\
\hline $22: 5 n-6$ & -0.03 & $-0.06,-0.01^{\star}$ & 0.02 & $-0.00,0.04$ & 0.05 & $0.02,0.07^{\star \star}$ & 0.04 & $0.01,0.07^{\star}$ \\
\hline$n-6$ LCPUFA & -0.85 & $-1.28,-0.43^{\star \star}$ & 0.23 & $-0.20,0.66$ & 1.05 & $0.57,1.53^{\star \star}$ & 0.95 & $0.47,1.43^{\star *}$ \\
\hline$n-6$ Fatty acids & 0.76 & $-0.15,1.67$ & 3.04 & $2 \cdot 12,3 \cdot 96^{\star \star}$ & $4 \cdot 14$ & $3 \cdot 11,5 \cdot 17^{\star \star}$ & $3 \cdot 13$ & $2 \cdot 10,4 \cdot 16^{\star \star}$ \\
\hline \multicolumn{9}{|l|}{$n-3$ PUFA } \\
\hline ALA $(18: 3 n-3)$ & -0.02 & $-0.04,0.01$ & 0.01 & $-0.02,0.03$ & 0.00 & $-0.03,0.03$ & 0.03 & $0.00,0.05^{\star}$ \\
\hline EPA $(20: 5 n-3)$ & -0.05 & $-0.07,-0.03^{\star \star}$ & -0.04 & $-0.06,-0.02^{\star \star}$ & -0.04 & $-0.06,-0.01^{*}$ & -0.03 & $-0.05,-0.01^{*}$ \\
\hline $22: 5 n-3$ & -0.09 & $-0.13,-0.05^{\star \star}$ & -0.06 & $-0.10,-0.02^{\star}$ & -0.05 & $-0.09,-0.00^{\star}$ & -0.04 & $-0.08,0.00$ \\
\hline DHA $(22: 6 n-3)$ & -0.22 & $-0.36,-0.07^{\star}$ & -0.11 & $-0.26,0.04$ & -0.15 & $-0.31,0.02$ & -0.10 & $-0.26,0.06$ \\
\hline$n-3$ LCPUFA & -0.36 & $-0.54,-0.18^{\star \star}$ & -0.21 & $-0.40,-0.03^{*}$ & -0.22 & $-0.42,-0.01^{*}$ & -0.16 & $-0.36,0.05$ \\
\hline$n-3$ Fatty acids & -0.38 & $-0.56,-0.19^{\star \star}$ & -0.21 & $-0.39,-0.02^{*}$ & -0.22 & $-0.43,-0.01^{\star}$ & -0.13 & $-0.34,0.08$ \\
\hline PUFA & 0.38 & $-0.63,1.39$ & 2.84 & $1 \cdot 81,3 \cdot 86^{\star \star}$ & 3.93 & $2 \cdot 78,5 \cdot 07^{\star \star}$ & 3.00 & $1 \cdot 86,4 \cdot 14^{\star *}$ \\
\hline Total fatty acid concentration $(\mu \mathrm{g} / 100 \mu \mathrm{l})$ & 35.5 & $12 \cdot 5,58 \cdot 5^{\star}$ & $20 \cdot 6$ & $-2 \cdot 6,43 \cdot 9$ & $-29 \cdot 9$ & $-55 \cdot 1,-3 \cdot 9^{\star}$ & $-30 \cdot 1$ & $-56 \cdot 0,-4 \cdot 2^{\star}$ \\
\hline$n-6: n-3$ PUFA ratio & $2 \cdot 3$ & $1 \cdot 5,3 \cdot 1^{\star \star}$ & $2 \cdot 7$ & $1 \cdot 9,3 \cdot 5^{\star \star}$ & 3.4 & $2 \cdot 5,4 \cdot 3^{\star \star}$ & 2.6 & $1 \cdot 7,3 \cdot 5^{\star \star}$ \\
\hline Mead acid:AA ratio & 0.01 & $0.00,0.02^{*}$ & 0.01 & $0.00,0.02^{*}$ & -0.00 & $-0.01,0.01$ & -0.00 & $-0.01,0.01$ \\
\hline EPA:AA ratio & -0.01 & $-0.01,-0.00^{\star \star}$ & -0.01 & $-0.01,-0.01^{\star *}$ & -0.01 & $-0.02,-0.01^{\star *}$ & -0.01 & $-0.01,-0.01^{* *}$ \\
\hline 22:5n-6:DHA ratio & 0.01 & $-0.02,0.04$ & 0.04 & $0.02,0.07^{\star}$ & 0.09 & $0.06,0.12^{\star \star}$ & 0.07 & $0.04,0.10^{\star \star}$ \\
\hline
\end{tabular}

$n$, Number of children in whom information was available; LA, linoleic acid; AA, arachidonic acid; LCPUFA, long-chain PUFA; $n-6: n-3$ PUFA ratio, ratio of total $n-6$ PUFA:total $n-3$ PUFA.

* $P<0.05$ and ${ }^{* *} P<0.001$ relative to admission values.

$\dagger$ Paired $t$ test analyses were done to test for differences in fatty acids at different times of follow-up compared with admission values.

was associated with a higher increase in LA values $(P=0.021)$, whereas an increase in height was associated with a smaller increase in LA $(P=0.043)$. Breast-fed children had a smaller increase in LA $(P<0.001)$ and a smaller decrease in EPA $(P=0.043)$ compared with non-breast-fed children. Children from families with recent fish intake had a smaller increase in $n$-6 LCPUFA $(P=0.045)$ and a greater decrease in $n$-3 LCPUFA values $(P=0.045)$. At discharge, children admitted with oedema had a smaller decrease in $n-3$ DPA than those without oedema $(P=0.038)$. High $\mathrm{Hb}$ values on admission predicted a higher increase in LA $(P=0.028)$ but a smaller increase in $n$-6 LCPUFA values $(P=0 \cdot 042)$. HIV-infected children had a smaller increase in LA values than those not infected $(P=0.001)$. CRP $>5 \mathrm{mg} / \mathrm{l}$ predicted a more pronounced increase in LA $(P=0.049)$ and a smaller decrease in $n$-3 LCPUFA values $(P=0 \cdot 031)$. Children in whom fever was reported at admission also had a greater increase in LA values compared with those without fever $(P=0.033)$

Table 5 represents a summary of predictors of changes in fatty acid values from admission to 8 weeks of follow-up in children with SAM. Boys had a smaller decrease in values of $n-3$ LCPUFA than girls $(P=0 \cdot 016)$. Weight gain at 8 weeks of follow-up was associated with a higher increase in LA values $(P=0.049)$, whereas an increase in height was associated with a smaller decrease in DHA values $(P=0 \cdot 036)$. Breast-fed children had a smaller decrease in $n-3$ LCPUFA compared with nonbreast-fed children $(P=0 \cdot 013)$. High $\mathrm{Hb}$ concentration was associated with a greater decrease in DHA values $(P=0.044)$. 

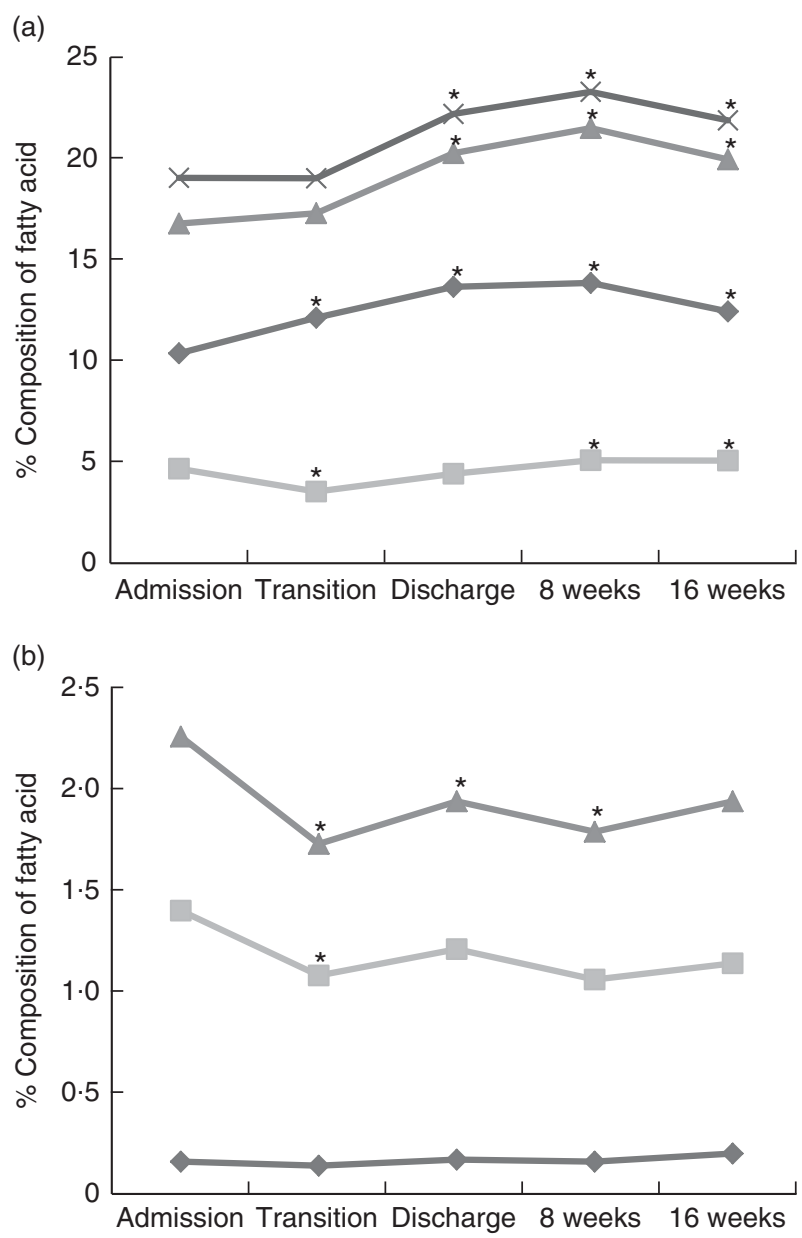

Fig. 1. Trend of changes in fatty acid values during treatment. (a) linoleic acid; -- , arachidonic acid; $-\leftarrow$, total $n-6$ PUFA; $\leftarrow$, total PUFA. (b) $a$-Linolenic acid; $\longrightarrow$, DHA; $-\longrightarrow$, total $n-3$ PUFA $-\leftarrow$. * Significant increase or decrease from admission values.

Children with oral thrush had a smaller increase in $n-6$ LCPUFA $(P=0.035)$, whereas those who had NG tube feeding in the first $2 \mathrm{~d}$ of admission had a smaller increase in $n-6$ LCPUFA $(P=0.027)$ and a greater decrease in $n-3$ LCPUFA $(P=0.047)$.

Children who died or were otherwise lost to follow-up had slightly higher CRP values on admission than those who remained in the study $(P=0.068)$, and were not different with respect to age, sex, breast-feeding, nutritional status and admission $\mathrm{Hb}$ concentration (data not shown). Admission values of LA, ALA, AA and DHA were also not different in children who were followed up and those not followed up.

\section{Discussion}

This study shows that in-hospital treatment of SAM with conventional F-75 and F-100 was associated with decreases in whole-blood n-3 LCPUFA proportions at discharge. As we demonstrate that the children with SAM already at admission had significantly lower proportions of LCPUFA than controls, this is a cause for concern. There could be many reasons for decreases in $n$ - 3 LCPUFA, one being that F-75 and F-100 contain

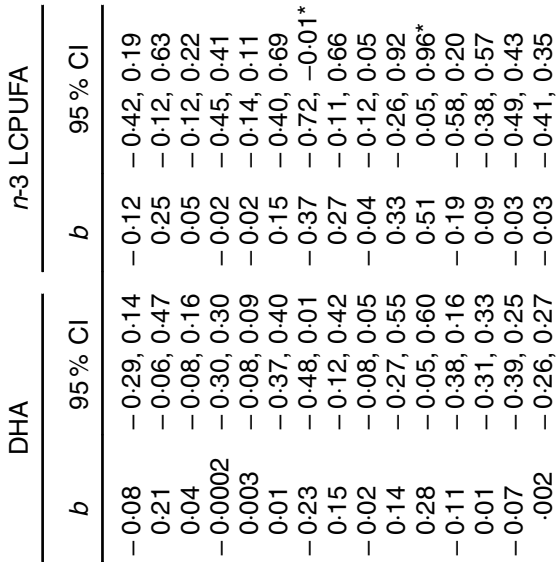

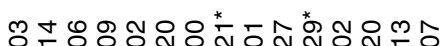

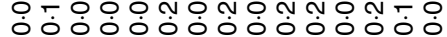

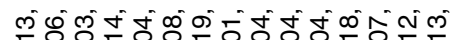

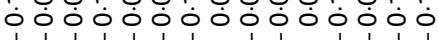

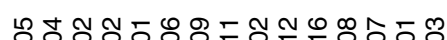

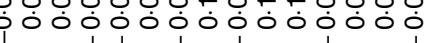

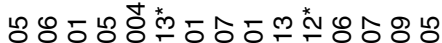

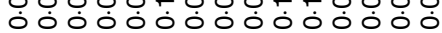

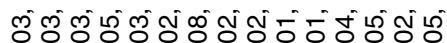

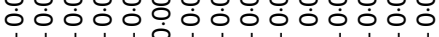

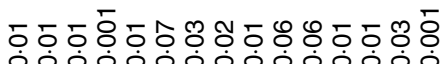

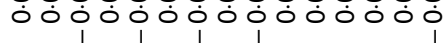

以ำ مَ-

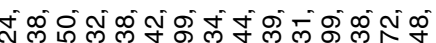

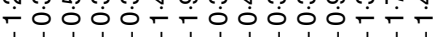
ᄂ

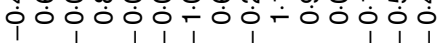

Oัن

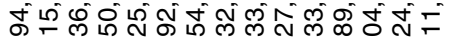
i o i o o

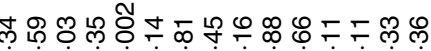
1000000000000́

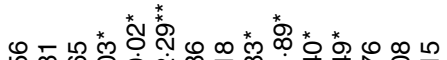

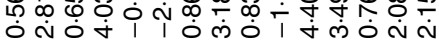

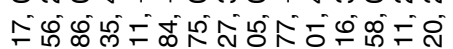

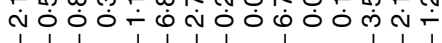

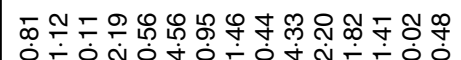
0

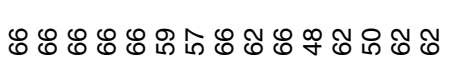

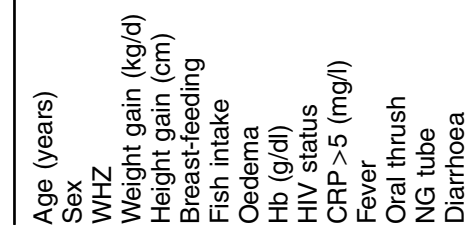


low levels of ALA ( 0.25 percentage of energy intake (\%E) and $0.3 \% \mathrm{E}$, respectively). Another reason may be that $\mathrm{F}-75$ and F-100 contain only ALA and LA and thus require an ability in the child to convert these into the functionally important LCPUFA, AA, EPA and DHA. Several studies suggest a compromised ability to elongate and desaturate in children with $\mathrm{SAM}^{(5,25)}$. Most recently, a study demonstrated that Kenyan children could only improve their DHA status when fish oil was added directly to the feeds, not with extra ALA ${ }^{(8)}$. Another important observation in this study was that the children with SAM had persistent lower whole-blood levels of PUFA, especially of $n-3$ PUFA, during recovery compared with healthy control children. The treatment resulted in normalisation in the percentage contribution of LA at discharge, but LA values had decreased to less than the control values again by 16 weeks of follow-up. The mean values of AA initially reduced at transition, but then increased during treatment, although it remained lower than that of control children at the end of recovery. These data agree with Koletzko et $a l .{ }^{(25)}$, who also found increasing levels of LA in Nigerian children with SAM and decreasing AA levels during treatment. In the current study, DHA values decreased during the entire follow-up period. Similarly, other studies have found a lack of increases in DHA values during dietary intervention of malnourished children ${ }^{(6,25)}$.

The decrease in $n$-3 LCPUFA was less pronounced in breastfed children. Breastmilk is a recognised source of $n-3$ LCPUFA, the composition being dependent on maternal nutrition ${ }^{(26,27)}$. Children from families with recent fish intake had a smaller increase in $n$-6 LCPUFA and a greater decrease in $n$ - 3 LCPUFA during treatment. This may imply that they had better LCPUFA levels at admission probably because of a better supply of LCPUFA than the therapeutic feeds given at the hospital. Furthermore, the changes in LCPUFA levels ceased to be significant at 8 weeks of follow-up, which may be related to a gradual return to a home diet. This emphasizes a need to revisit the PUFA content in therapeutic feeds.

Children with lower $\mathrm{Hb}$ at admission had a less pronounced increase in LA at discharge and a higher increase in total $n-6$ LCPUFA. We anticipate that there may be a build-up of $\mathrm{Hb}$ and erythrocytes during rehabilitation. It could be speculated that the increased dietary supply of LA during treatment may have been converted to $n$-6 LCPUFA for deposition in the erythrocyte cell membrane as SAM subsides and growth and build of tissues restarts. Hence, the increase in whole-blood $n-6$ LCPUFA may be more pronounced in anaemic children in an effort to restore $\mathrm{Hb}$ levels. Children infected with HIV also had a smaller increase in LA by the time of discharge than HIV-negative children. Low levels of LA have previously been described in HIV-infected children, and they have been attributed to high PUFA turn-over ${ }^{(28)}$.

A number of children with SAM have infections when first admitted $^{(29,30)}$, and they all receive empirical antibiotics according to guidelines ${ }^{(10)}$. Infections in general, as indicated by $\mathrm{CRP}>5 \mathrm{mg} / \mathrm{l}$ at admission, predicted a more pronounced increase in whole-blood LA values and a smaller decrease in the level of $n$-3 LCPUFA at discharge, but the association ceased to be significant by 8 weeks of follow-up. When infections are controlled, the need for AA for eicosanoid synthesis may 
decrease, which could explain this observed increase in LA values. In addition, as inflammatory processes subside, it is plausible that less $n$ - 3 LCPUFA is catabolised for the production of anti-inflammatory mediators, which may explain the observed smaller decrease in $n$-3 LCPUFA. However, children with oral thrush at admission and those who had been fed through the NG tube within the first $2 \mathrm{~d}$ of admission had a smaller increase in values of AA and total $n$ - 6 LCPUFA at 8 weeks of follow-up and NG tube fed children also had a larger decrease in DHA and total $n-3$ LCPUFA values at 8 weeks of follow-up. Oral thrush is a fungal infection of oral cavity mucous membrane, and children who were NG tube fed were quite sick and prone to severe infections. All these processes could perhaps lead to marked derangement in metabolism, increased production of inflammatory eicosanoids and thus contribute to the use of LCPUFA.

One limitation to this study is its observational nature limiting causal inference of the decreased values of LCPUFA in children with SAM. Second, as the aim was to explore predictors of changes in essential fatty acids, a large number of tests were performed, which may have increased the risk of chance findings. Furthermore, we had a high loss of patients, particularly at 8 and 16 weeks of follow-up, and as many of these dropouts were because of children dying, it is likely that the children lost were sicker than those who remained in the study. However, when assessing changes over time, we only compared the children for whom data were available at both admission and later. The high loss to follow-up of children reduces the statistical power of our study. Besides loosing children in follow-up, it was not possible to get blood samples from all the children we were able to follow-up. In addition, whole-blood fatty acid analysis limits our ability to determine fatty acid distribution in the different lipid classes as compared with detailed sub analyses. Having said this, our results are in line with previous results. Another limitation may be that control children were only studied once, whereas study children were followed up over time. It is possible that some change would also have occurred in the fatty acid proportions in control children over time, because of the seasonal change in diet. However, the change in study children during in-patient treatment is unlikely to be explained by seasonal variation, as the diet was standardised, with almost no local foods included.

There is an increasing concern whether therapeutic feeds are able to correct essential fatty acid status of children recovering from $\mathrm{SAM}^{(31)}$. As the children were treated according to the Uganda Ministry of Health guidelines, PUFA levels at transition reflect the impact of F-75, at discharge the impact of F-100 and at 8 weeks of follow-up the impact of RUTF. During recovery, energy- and nutrient-dense foods are encouraged at home to achieve good catch-up growth; hence, 16 weeks of follow-up reflects the impact of the home diet. Thus, the low levels of particularly $n$-3 LCPUFA at discharge and 8 weeks of recovery from SAM suggest that the F-100 and RUTF cannot correct preexisting compromised essential fatty acid status. Furthermore, the ratio of $n-6: n-3$ PUFA at admission was lower in children with SAM, but then became higher than in the healthy controls following treatment, thus reflecting an overall pronounced lack of $n-3$ PUFA in all therapeutic diets. For RUTF, WHO and the
World Food Programme recommend a fat content of $45-60 \% \mathrm{E}$, of which $3-10 \% \mathrm{E}$ should come from $n-6$ fatty acids and $0 \cdot 3-2 \cdot 5 \% \mathrm{E}$ from $n-3$ fatty acids $^{(11)}$. F-100 follows these same specifications for essential fatty acids. Standard RUTF contains ALA corresponding to $0 \cdot 3-2 \cdot 5 \% \mathrm{E}$ and $3-10 \% \mathrm{E}$ LA. Both RUTF and F-100 meet the WHO minimum recommendations for ALA and LA and none of the recommendations mention anything about content of LCPUFA relative to the shorter-chain PUFA in therapeutic diets.

Hsieh et $a l .{ }^{(9)}$ demonstrated a worsening of $n$-3 LCPUFA status in children with uncomplicated SAM after 4 weeks of treatment with conventional RUTF compared with children treated with HO-RUTF with decreased LA contents. Furthermore, Jones et al. ${ }^{(8)}$ noted that provision of RUTF with elevated ALA had minimal impact on $n-6$ and $n-3$ PUFA status, but that addition of fish oil was associated with marked increases in $n-3$ LCPUFA. HO-RUTF on the other hand led to relative increases of $+29 \%$ and $+87 \%$ for DHA and EPA, respectively ${ }^{(9)}$. The strategy of increasing oleic acid and decreasing LA to a 1:1 ratio with ALA may therefore be a promising way to improve the $n-3$ LCPUFA status of children treated for SAM without having to add fish oil to the therapeutic feeds.

\section{Conclusion}

Whole-blood $n$-6 PUFA proportions increased from admission over discharge to follow-up, except for AA, whereas $n$-3 LCPUFA proportions of EPA and DHA decreased during in-hospital treatment. Furthermore, both $n-3$ and n-6 PUFA proportions remained lower than those of the healthy controls at the end of treatment. Thus, the current recommended therapeutic feeds for rehabilitating children with SAM are not able to correct their whole-blood LCPUFA compromised status. Although there were significant increases in $n-6$ LCPUFA values during treatment, the decreases in $n-3$ LCPUFA and AA proportions from admission to discharge pose particular concern, as AA and DHA play important functional roles in the brain, retina and immune system. F-75 and F-100 formulations with higher $n-3$ PUFA contents to decrease $n-6: n-3$ ratio and preformed LCPUFA may need to be considered in therapeutic diets for children with SAM.

\section{Acknowledgements}

The authors are grateful to Elizabeth Kiboneka, head of Mwanamugimu Nutrition Unit, for guidance and facilitating the study; to Sofine Heilskov, Amira Catharina Khatar Sørensen and Kia Hee Schultz for data collection; to Julian Eyotaru, Loice Atuhaire, Susan Awori, Justine Naggayi and Joseph Mbabazi for data collection and skilled care of the patients; and to Christian Ritz for statistical help.

The study was funded by a PhD grant from University of Copenhagen, and received support from Augustinus Fonden, Brødrene Hartmanns Fond, Arvid Nielsens Fond, Axel Muusfeldts Fond, Aase and Einar Danielsens Fond and Torkild Steenbecks Legat. The funding sources had no influence on design of the study, data collection and analysis, or interpretation of the results. 
H. F., M. J. H. R., C. G. M. and H. N. designed the study; E. B.-I., M. J. H. R. and C. G. M. collected data; E. B.-I. analyzed data and drafted the initial manuscript; L. L. contributed to the interpretation of the data; E. B.-I. had primary responsibility for final content. All above authors plus E. M., K. F. M., A. B. and K. D. S. reviewed and revised the manuscript. All authors read and approved the final manuscript.

The authors declare that there are no conflicts of interest.

\section{References}

1. Black RE, Victora CG, Walker SP, et al. (2013) Maternal and child undernutrition and overweight in low-income and middle-income countries. Lancet 382, 427-451.

2. World Health Organization (2003) Guidelines for the Inpatient Treatment of Severely Malnourished Children. Geneva: WHO. http://www.who.int/nutrition/publications/severemalnutrition/ 9241546093/en/ (accessed February 2015).

3. World Health Organization, WFP, SCN, et al. (2007) Joint Statement on Community-Based Management of Severe Acute Malnutrition. Geneva: UNICEF Publications. http://www.unicef. org/publications/index_39468.html (accessed June 2015).

4. World Health Organization (2007) Community-Based Management of Severe Acute Malnutrition. Geneva: WHO. http://www.who.int/nutrition/topics/statement_commbased_ malnutrition/en/ (accessed June 2015).

5. Lauritzen L, Hansen HS, Jorgensen MH, et al. (2001) The essentiality of long chain $n$-3 fatty acids in relation to development and function of the brain and retina. Prog Lipid Res 40, 1-94.

6. Smit E, Muskiet F \& Boersma E (2004) The possible role of essential fatty acids in the pathophysiology of malnutrition: a review. Prostaglandins Leukot Essent Fatty Acids 71, 241-250.

7. Decsi T \& Koletzko B (2000) Effects of protein-energy malnutrition and human immunodeficiency virus-1 infection on essential fatty acid metabolism in children. Nutrition 16, $447-453$.

8. Jones KD, Ali R, Khasira MA, et al. (2015) Ready-to-use therapeutic food with elevated $n-3$ polyunsaturated fatty acid content, with or without fish oil, to treat severe acute malnutrition: a randomized controlled trial. BMC Med 13, 93.

9. Hsieh J-C, Liu L, Zeilani M, et al. (2015) High oleic ready-touse therapeutic food maintains docosahexaenoic acid status in severe malnutrition: a randomized, blinded trial. $J$ Pediatr Gastroenterol Nutr 61, 138-143.

10. Ministry of Health, Uganda (2010) Integrated Management of Acute Malnutrition Guidelines. Kampala: Ministry of Health.

11. World Health Organization (2007) Joint Statement on the Community-Based Management of Severe Acute Malnutrition. Geneva: WHO. http://www.who.int/maternal_child_adolescent/ documents/a91065/en/ (accessed August 2014).

12. World Health Organization \& United Nations Children's Fund (2009) WHO Child Growth Standards and the Identification of Severe Acute Malnutrition in Infants and Children - A Joint Statement. Geneva: WHO.

13. World Health Organization (2012) Service Delivery Approaches to HIV Testing and Counselling (HTC): A Strategic HTC Policy Framework. Geneva: WHO Press.

14. Namusoke H, Hother AL, Rytter MJ, et al. (2016) Changes in plasma phosphate during in-patient treatment of children with severe acute malnutrition: an observational study. Am J Clin Nutr 103, 551-558.
15. World Health Organization (2009) WHO Child Growth Standards and the Identification of Severe Acute Malnutrition in Infants and Children. Geneva: WHO. http://www.who.int/ nutrition/publications/severemalnutrition/9789241598163/en/ (accessed February 2015).

16. Metherel AH \& Stark KD (2015) Cryopreservation prevents iron-initiated highly unsaturated fatty acid loss during storage of human blood on chromatography paper at $-20^{\circ} \mathrm{C}$. J Nutr 145, 654-660

17. Marangoni F, Colombo C \& Galli C (2004) A method for the direct evaluation of the fatty acid status in a drop of blood from a fingertip in humans: applicability to nutritional and epidemiological studies. Anal Biochem 326, 267-272.

18. Metherel AH, Aristizabal Henao JJ \& Stark KD (2013) EPA and DHA levels in whole blood decrease more rapidly when stored at $-20^{\circ} \mathrm{C}$ as compared with room temperature, 4 and $-75^{\circ} \mathrm{C}$. Lipids 48, 1079-1091.

19. Galli C, Rise P, Ghezzi S, et al. (2009) Fast determination of fatty acids in whole blood collected from fingertips: application to the assessment of fatty acid patterns (and various indexes) in population studies. World Rev Nutr Diet 100, 35-45.

20. Kume A, Miyazaki T, Kitamura Y, et al. (2008) High levels of saturated very long-chain fatty acid (hexacosanoic acid; C26:0) in whole blood are associated with metabolic syndrome in Japanese men. Diabetes Res Clin Pract 80, 259-264.

21. Armstrong J, Metherel A \& Stark K (2008) Direct microwave transesterification of fingertip prick blood samples for fatty acid determinations. Lipids 43, 187-196.

22. Metherel AH, Taha AY, Izadi H, et al. (2009) The application of ultrasound energy to increase lipid extraction throughput of solid matrix samples (flaxseed). Prostaglandins Leukot Essent Fatty Acids 81, 417-423.

23. Rytter MJ, Namusoke H, Babirekere-Iriso E, et al. (2015) Social, dietary and clinical correlates of oedema in children with severe acute malnutrition: a cross-sectional study. $B M C$ Pediatr 15, 25.

24. Heilskov S, Vestergaard C, Babirekere E, et al. (2015) Characterisation and scoring of skin changes in severe acute malnutrition in children between 6 months and 5 years of age. J Eur Acad Dermatol Venereol 29, 2463-2469.

25. Koletzko B, Abiodun P, Laryea M, et al. (1986) Fatty acid composition of plasma lipids in Nigerian children with protein-energy malnutrition. Eur J Pediatr 145, 109-115.

26. Urwin HJ, Zhang J, Gao Y, et al. (2013) Immune factors and fatty acid composition in human milk from river/lake, coastal and inland regions of China. J Nutr 109, 1949-1961.

27. Smit EN, Koopmann M, Boersma ER, et al. (2000) Effect of supplementation of arachidonic acid (AA) or a combination of AA plus docosahexaenoic acid on breastmilk fatty acid composition. Prostaglandins Leukot Essent Fatty Acids $\mathbf{6 2}$, 335-340.

28. Agostoni C, Riva E, Esposito S, et al. (2000) Fatty acid composition of plasma lipids in HIV-infected children. Comparison with seroreverters. Acta Paediatr 89, 172-176.

29. Babirekere-Iriso E, Musoke P \& Kekitiinwa A (2006) Bacteraemia in severely malnourished children in an HIV-endemic setting. Ann Trop Paediatr 26, 319-328.

30. Bachou H, Tylleskär T, Kaddu-Mulindwa DH, et al. (2006) Bacteraemia among severely malnourished children infected and uninfected with the human immunodeficiency virus-1 in Kampala, Uganda. BMC Infect Dis 6, 160.

31. Brenna JT, Akomo P, Bahwere P, et al. (2015) Balancing omega- 6 and omega- 3 fatty acids in ready-to-use therapeutic foods (RUTF). BMC Med 13, 117. 